\title{
Testing Inflation: A Bootstrap Approach
}

\author{
Latham Boyle $^{1}$ and Paul J. Steinhardt ${ }^{2,3}$ \\ ${ }^{1}$ Canadian Institute for Theoretical Astrophysics (CITA), Toronto M5S 3H8, Canada \\ ${ }^{2}$ Princeton Center for Theoretical Science, Princeton University, Princeton, New Jersey 08544, USA \\ ${ }^{3}$ Department of Physics, Princeton University, Princeton, New Jersey 08544, USA
}

(Received 15 October 2008; revised manuscript received 6 September 2010; published 6 December 2010)

\begin{abstract}
We note that the essential idea of inflation, that the Universe underwent a brief period of accelerated expansion followed by a long period of decelerated expansion, can be encapsulated in a "closure condition" which relates the amount of accelerated expansion during inflation to the amount of decelerated expansion afterward. We present a protocol for systematically testing the validity of this condition observationally.
\end{abstract}

DOI: 10.1103/PhysRevLett.105.241301

PACS numbers: 98.80.Cq, 98.80.Es

Is it possible to show convincingly that inflation $[1,2]$ is responsible for the large-scale homogeneity, isotropy, and flatness of the Universe, and the primordial spectrum of metric fluctuations that seeded galaxy formation and sourced the temperature and polarization variations in the cosmic microwave background (CMB)? Some would claim no, because there is too much freedom in constructing inflationary models. Even if one shows that the observations are consistent with the predictions of a particular inflationary model, this is unconvincing because, for virtually any given combination of observations, one can design many inflationary models that reproduce them. If a theory allows everything, it has no predictive power.

In this Letter, though, we describe how to combine observations into a sequence of "bootstrap tests" that, if any one of them is passed, will be the most direct confirmation possible that the Universe underwent a brief period of acceleration $\left(d^{2} a / d t^{2}>0\right)$ followed by a long period of deceleration $\left(d^{2} a / d t^{2}<0\right)$. [Here $a(t)$ is the Robertson-Walker scale factor and $H(t) \equiv d(\ln a) / d t$ is the Hubble rate [2].] Let us briefly summarize the basic idea, with details postponed until the next section. First, note that $a(t) H(t)$ grows during acceleration, and shrinks during deceleration. According to the inflationary scenario [2], any observable Fourier mode of the cosmological density field with comoving wave number $k_{*}$ reached a moment during inflation known as "horizon exit," at which the ratio $a(t) H(t) / k_{*}$ was unity; then, during the period remaining before the end of inflation, this ratio grew by $N_{\text {bef }}\left(k_{*}\right) e$ folds; and finally, after inflation, the ratio shrunk by $N_{\text {aft }} e$ folds, reaching the value of $a_{0} H_{0} / k_{*}<1$ measured today. Thus, in this picture, the mode $k_{*}$ must satisfy the "closure condition"

$$
\ln \left(a_{0} H_{0} / k_{*}\right)=N_{\text {bef }}\left(k_{*}\right)-N_{\text {aft }} .
$$

How can we test this equation? If we know the temporal evolution of $H$, from the moment that $k_{*}$ leaves the horizon until the end of inflation, we have enough information to compute $N_{\text {bef }}\left(k_{*}\right)$ and $N_{\text {aft }}$ and check that Eq. (1) is correct.
Unfortunately, cosmological observations will never give us this much information; instead, assuming inflation is correct, they will provide the first few terms in a Taylor approximation to $H$ around the moment that the "WMAP wave number" [3] $k_{*}=0.002 / \mathrm{Mpc}$ left the horizon. Imagine we only have enough observations to determine this Taylor approximation up to $j$ th order: this is our best guess for $H$ given the available data. Under the assumption that this guess remains valid all the way to the end of inflation, we can check whether Eq. (1) is true. If it is, then we not only have evidence for our guess, but for the idea of inflation on which it is based: we have pulled ourselves up by our bootstraps. Note that $a_{0} H_{0} / k_{*}$, as measured today, is exponentially sensitive to both $N_{\text {bef }}\left(k_{*}\right)$, which depends on the expansion history during inflation, and $N_{\text {aft }}$, which depends on the expansion history after inflation. Thus, if observations can be used to determine all three quantities and if they are shown to satisfy the closure condition, even an ardent skeptic would be hard pressed to discount it as coincidence; it would be strong evidence for inflation, and a tall challenge for any competing theory. And if there are any doubters, the bootstrap method offers in some cases a series of follow-up checks that can turn a convincing verification into an overwhelming one.

As discussed in the conclusion, the closure test also has the advantage that it is experimentally easier to apply compared to other proposed inflationary tests, such as the "consistency relations" [4,5]. As for failing the closure test, this does not mean inflation is ruled out, because it is always possible to construct inflationary models that fit the data; as precision improves, observers can continue to distinguish viable and nonviable models. However, in this sad circumstance, cosmological observations will probably never yield the kind of convincing confirmation of inflation discussed here.

Key observables and equations.-Before describing the bootstrap tests, let us introduce the key observables, equations and parameters we will need. From $\Delta_{\mathcal{R}}^{2}(k)$ and $\Delta_{h}^{2}(k)$, 
the scalar and tensor power spectra, one defines the tensor to scalar ratio $r \equiv \Delta_{h}^{2} / \Delta_{\mathcal{R}}^{2}$, the scalar index $n_{s}-1 \equiv$ $d\left(\ln \Delta_{\mathcal{R}}^{2}\right) / d(\ln k)$, its "running" $\alpha_{s} \equiv d n_{s} / d(\ln k)$, the "running of the running" $\beta_{s} \equiv d \alpha_{s} / d(\ln k)$, and so on, all measured at the wave number $k_{*}$ at which they are most precisely determined. We follow the standard WMAP definitions for all of these observables [3], and for concreteness fix $k_{*}=0.002 / \mathrm{Mpc}$ following WMAP [3].

We focus first on the case where the Hubble expansion rate $H$ during inflation is governed by a single order parameter that acts just like a single inflaton scalar field, $\varphi$ [6-8] (Generalizations will be discussed in the concluding section.) For a clear derivation and presentation of all of the equations in this section, see Liddle et al. [6]. Subscripts "*" and "end" will be used to indicate that the corresponding quantity is to be evaluated at the moment when $k_{*}$ exits the horizon, or at the end of inflation, respectively. Without loss of generality, we can choose $\varphi_{*}=0$, and Taylor expand

$$
H(\varphi)=H_{*}+H_{*}^{\prime} \varphi+\frac{1}{2} H_{*}^{\prime \prime} \varphi^{2}+\frac{1}{6} H_{*}^{\prime \prime \prime} \varphi^{3}+\ldots
$$

If, again without loss of generality, we take $d \varphi / d t>0$ (or, equivalently, $H_{*}^{\prime}<0$ ), and choose "reduced Planck units" with $\hbar=c=8 \pi G=1$, then the first few coefficients are

$$
\begin{aligned}
H_{*} & =\frac{\pi\left(\Delta_{\mathcal{R}}^{2}\right)^{1 / 2}}{2}(2 r)^{1 / 2}, \\
H_{*}^{\prime} & =\frac{\pi\left(\Delta_{\mathcal{R}}^{2}\right)^{1 / 2}}{8}(-r), \\
H_{*}^{\prime \prime} & =\frac{\pi\left(\Delta_{\mathcal{R}}^{2}\right)^{1 / 2}}{32}(2 r)^{1 / 2}\left[r+4\left(n_{s}-1\right)\right], \\
H_{*}^{\prime \prime \prime} & =\frac{\pi\left(\Delta_{\mathcal{R}}^{2}\right)^{1 / 2}}{128}\left[64 \alpha_{s}-3 r^{2}-20 r\left(n_{s}-1\right)\right] .
\end{aligned}
$$

The end of inflation $\left(H=H_{\text {end }}\right.$ and $\left.\varphi=\varphi_{\text {end }}\right)$ occurs when $\ddot{a}=0$ or, equivalently,

$$
H_{\text {end }}=-\sqrt{2} H_{\text {end }}^{\prime} .
$$

Finally, in the closure condition (1), we have

$$
N_{\text {bef }}\left(k_{*}\right)=\ln \left(H_{\text {end }} / H_{*}\right)-\frac{1}{2} \int_{\varphi_{*}}^{\varphi_{\text {end }}} d \varphi\left[H(\varphi) / H^{\prime}(\varphi)\right]
$$

and

$$
N_{\text {aft }}=\ln \left[\Omega_{\text {rad }}^{1 / 4}\left(H_{\text {end }} / H_{0}\right)^{1 / 2}\right]+\Delta N,
$$

where $\Omega_{\mathrm{rad}}$ is the current ratio of the radiation density to the critical density and

$$
\Delta N \equiv(1 / 12)\left[\left(1-3 w_{\text {re }}\right) /\left(1+w_{\text {re }}\right)\right] \ln \left(\rho_{\text {re }} / \rho_{\text {end }}\right)
$$

represents the uncertain physics of the epoch between the end of inflation and the start of radiation domination: $w_{\text {re }}$ is the effective equation-of-state during this epoch, and $\rho_{\text {re }}$ is the energy density at the start of radiation domination. We first consider the case where $\Delta N \approx 0$, which corresponds to "efficient" reheating $\left(w_{\text {re }} \approx 1 / 3\right.$ or $\left.\rho_{\text {re }} \approx \rho_{\text {end }}\right)$.
However, the uncertainty in $\Delta N$, does not seriously interfere with the bootstrap test. To illustrate the point, we let $\Delta N$ be a free parameter, subject only to the weak assumptions that $0 \leq w_{\text {re }} \leq 1 / 3$ and $\rho_{\text {bbn }} \leq \rho_{\text {re }} \leq \rho_{\text {end }}$, where $\rho_{\text {bbn }} \approx(1 \mathrm{MeV})^{4}$ is roughly the energy density during big bang nucleosynthesis (BBN); then $\Delta N \leq 0$.

The bootstrap tests.-The bootstrap test uses precise observations at $k=k_{*}$ to obtain progessively better estimates of $H(\varphi)$ and $H_{\text {end }}$, which are, then, applied to determine if the closure condition is satisfied. If we regard $\Delta_{\mathcal{R}}^{2}=(2.45 \pm 0.1) \times 10^{-9}$ [3] as an already-measured quantity, then the Taylor expansion (2) and (3) of $H(\varphi)$ organizes the remaining observables into an ordered list $\left\{r, n_{s}, \alpha_{s}, \beta_{s}, \ldots\right\}$ in the sense that, if we imagine that we only know the first $j$ items in this list, then we can only determine the Taylor expansion up to $j$ th order [9]. This is the best guess for $H(\varphi)$ based on the available data; using it, $H_{\text {end }}$ is computed from Eq. (4); $N_{\text {bef }}\left(k_{*}\right)$ and $N_{\text {aft }}$ are determined from Eqs. (5) and (6); and, finally., the closure condition (1) is checked.

The $j$ th bootstrap test is satisfied if the first $j$ observables satisfy the closure condition. In practice, only the first three observables $\left\{r, n_{s}, \alpha_{s}\right\}$ can be detected or constrained tightly enough to be relevant for confirming inflation. Therefore, the first three bootstrap tests are the relevant ones, for all practical purposes: let us describe them and explain how they may be confirmed and cross-checked with forthcoming observations.

First bootstrap test.-To start, imagine we are only given the first observable, $r$, so our best guess for $H(\varphi)$ is $H_{*}+$ $H_{*}^{\prime} \varphi$. We introduce this into Eq. (4) to obtain $\varphi_{\text {end }}=$ $2^{1 / 2}\left[(16 / r)^{1 / 2}-1\right]$, and apply these expressions for $H(\varphi)$ and $\varphi_{\text {end }}$ to Eqs. (5) and (6) to obtain $N_{\text {bef }}\left(k_{*}\right)$ and $N_{\text {aft }}$. Then, the closure condition, Eq. (1), is satisfied if

$$
r(\Delta N)=8 /[A+\Delta N+1 / 2],
$$

where $\quad A \equiv \ln \left(a_{0} H_{0} / k_{*}\right)+\frac{1}{4} \ln \left(8 \Omega_{\mathrm{rad}} \pi^{2} \Delta_{\mathcal{R}}^{2} / H_{0}^{2}\right) \approx 61$. This corresponds to $r=0.13$ if $\Delta N=0$, and $0.13<r<$ 0.17 if the uncertainty in $\Delta N$ is included.

If observations pass this first bootstrap test, it will be a remarkable success for the inflationary paradigm, and one that can be checked: since true success should not be spoiled by the next observable, $n_{s}$, we expect $n_{s}=1-$ $r / 4$ (so that $H_{*}^{\prime \prime} \approx 0$ ). If this follow-up test is also successful, then it should not be spoiled by the next observable, $\alpha_{s}$ : thus we expect $\alpha_{s}=\left[3 r^{2}+20 r\left(n_{s}-1\right)\right] / 64$ (so that $H_{*}^{\prime \prime \prime} \approx 0$ ). If observations pass the first bootstrap test (1), plus the two follow-up tests, it will be overwhelming evidence for a period of inflationary expansion.

Second bootstrap test.-If the first bootstrap test fails, proceed to the second. Given the measured values of $\left\{r, n_{s}\right\}$, the best guess for $H(\varphi)$ is now $H_{*}+H_{*}^{\prime} \varphi+$ $\frac{1}{2} H_{*}^{\prime \prime} \varphi^{2}$. We introduce this expression into (4) and find the smallest positive root: $\varphi_{\text {end }}\left(r, n_{s}\right)$. Next these formulae for $H(\varphi)$ and $\varphi_{\text {end }}$ can be used in Eqs. (5) and (6) to find 
$N_{\text {bef }}\left(k_{*}\right)$ and $N_{\text {aft }}$. Finally, substituting all of this into the closure condition (1), we obtain a relation between $r, n_{s}$ corresponding to the solid black curve in Fig. 1; allowing for the uncertainty in $\Delta N$ thickens the curve to the shaded region in the figure. Passing the second bootstrap test would be nearly as remarkable as passing the first, and, here again, the success can be verified by measuring the next observable, $\alpha_{s}$, and finding that $\alpha_{s}=\left[3 r^{2}+\right.$ $\left.20 r\left(n_{s}-1\right)\right] / 64$ (so that $H_{*}^{\prime \prime \prime} \approx 0$ ).

Third bootstrap test.- - If the first two bootstrap tests fail, proceed to next order. Use the first three observables $\left\{r, n_{s}, \alpha_{s}\right\}$ to determine the coefficients in $H(\varphi)=H_{*}+$ $H_{*}^{\prime} \varphi+\frac{1}{2} H_{*}^{\prime \prime} \varphi^{2}+\frac{1}{6} H_{*}^{\prime \prime \prime} \varphi^{3}$. Introduce this into Eq. (4) to find the smallest positive root $\varphi_{\text {end }}\left(r, n_{s}, \alpha_{s}\right)$ and use the expressions for $H(\varphi)$ and $\varphi_{\text {end }}$ in Eqs. (5) and (6), to compute $N_{\text {bef }}\left(k_{*}\right)$ and $N_{\text {aft }}$. Finally, substitute these expressions into Eq. (1) to check the closure condition. If $\Delta N=0$, the closure condition will be satisfied for a twodimensional surface in the three-dimensional space parametrized by $\left\{r, n_{s}, \alpha_{s}\right\}$ : several surface contours are shown in Fig. 2. Allowing for the uncertainty in $\Delta N$ thickens this surface into a "thin slab" (or, equivalently, each curve in Fig. 2 extends downward to form a strip). In the limit of extremely small $r$, this slab has a simple analytic description: $\varphi_{\text {end }}\left(r, n_{s}, \alpha_{s}\right)$ is given by $\varphi_{\text {end }}=$ $\left(-2 / \alpha_{s}\right)^{1 / 2} r^{1 / 4}$, and $r=r\left(n_{s}, \alpha_{s}, \Delta N\right)$ satisfies

$$
r=8 \exp \left[\frac{8 \pi-16 \arctan \left[\left(1-n_{s}\right) / y\right]}{y}-4(A+\Delta N)\right]
$$

where $y \equiv\left[-4 \alpha_{s}-\left(n_{s}-1\right)^{2}\right]^{1 / 2}$.

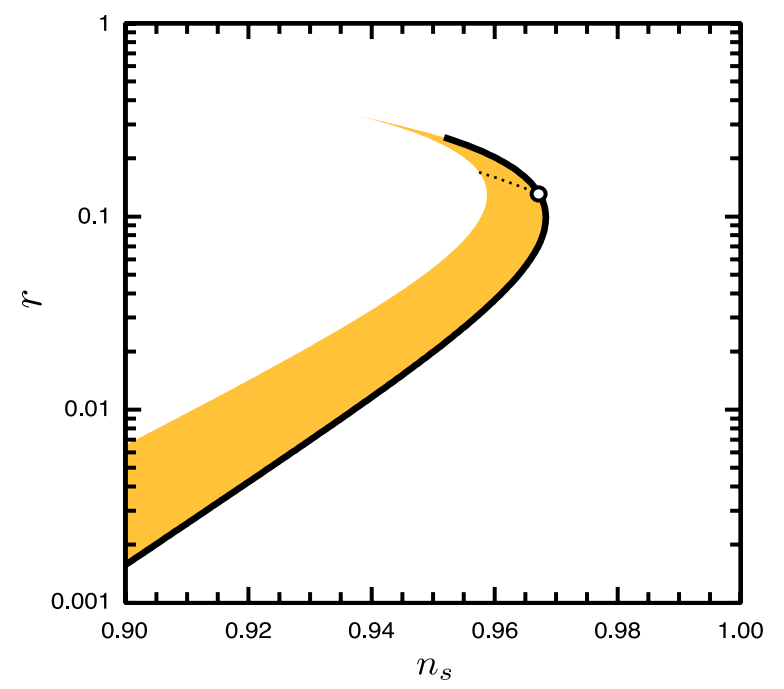

FIG. 1 (color online). First and second bootstrap tests: The first test is satisfied for $r$ and $n_{s}$ corresponding to the open white circle for efficient reheating; the circle becomes a short arc (dotted) if one includes the uncertainty in the "reheating epoch" (parameterized by $\Delta N$ ). The second test is passed if $r$ and $n_{s}$ lie anywhere along the solid black curve or, allowing for the uncertainty in $\Delta N$, the black curve plus shaded region.
We can make $\alpha_{s}$ as negative as possible (for fixed $n_{s}$ ) by first letting $r$ be as small as possible [for illustration, let us take the relatively weak assumption $\rho_{f}>(1 \mathrm{TeV})^{4}$ and hence $r>8 \times 10^{-55}$ ], and then letting $\Delta N$ be as negative as possible $\left(w_{\text {re }}=0\right.$ and $\left.\rho_{\text {re }}=\rho_{\text {bbn }}\right)$. In this way, we find that, if $\left\{r, n_{s}, \alpha_{s}\right\}$ pass the third bootstrap test, then $\alpha_{s}$ has a lower bound [10] $\alpha_{s}>\alpha_{s}^{\min }\left(n_{s}\right)$, where $\alpha_{s}^{\min }\left(n_{s}\right)$ varies smoothly from $\alpha_{s}^{\min }=-0.0094$ (for $n_{s}=0.9$ ) to $\alpha_{s}^{\min }=$ -0.0161 (for $\left.n_{s}=1\right)$.

We have seen that, if the first bootstrap test is passed, then $n_{s}$ and $\alpha_{s}$ provide two cross-checks, and, similarly, if the second bootstrap is passed, then $\alpha_{s}$ provides a single cross-check. But if the third bootstrap relation correctly predicts that the $\left\{r, n_{s}, \alpha_{s}\right\}$ lie in the slab described above, there will be no analogous cross-checks available, since we will have used up our observables (see below for caveats). Nevertheless, passing the third test is an impressive verification of the inflationary principle.

Discussion.-Observations will measure $r$ and $n_{s}$ to a precision of roughly \pm 0.01 in this decade, and perhaps \pm 0.001 eventually. The first bootstrap test would give the most impressive proof of inflation, since it makes the largest number of verifiable follow-up predictions; the ranges $0.13<r<0.17$ and $n_{s}=1-r / 4$ still agree well with current observations [3], but will either be confirmed or ruled out within the next few years. If the first bootstrap test fails, the second may be passed for a wider range of $r$, but since it relates $r$ to $n_{s}$, the allowed range of $r$ may be restricted by constraining $n_{s}$. For example, if $n_{s}>0.94$, as suggested by WMAP5 [3], then the second bootstrap test only requires searching for $r>0.01$, and thus may also be completed over the coming decade. And then, if CMB

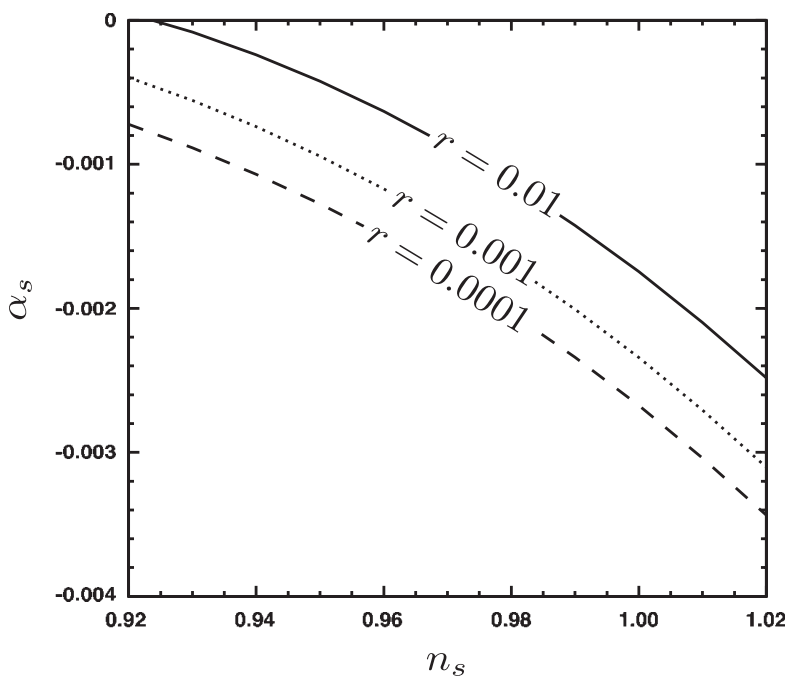

FIG. 2. The third bootstrap relation constrains $\left(r, n_{s}, \alpha_{s}\right)$ to lie on a curve, as shown, assuming efficient reheating $(\Delta N=0)$; including this uncertainty extends each curve downward (towards more easily observable values of $\alpha_{s}$ ) into a strip of finite thickness. Note that these curves include values of $\left(r, n_{s}\right)$ that fail the first two bootstrap tests. 
polarization experiments determine that $r<0.01$, all is not lost: the third bootstrap test may still be passed, but only if $\alpha_{s}$ has a substantially negative value (see Fig. 2)-e.g., negative enough to be detected by a proposed high-redshift galaxy survey designed to measure $\left|\alpha_{s}\right| \sim 0.001$ [11]. But if $\alpha_{s}$ is too negative, all three bootstrap tests fail: e.g., if $\alpha_{s}<-0.016$, then the tests fail for all $n_{s}<1$, according to the discussion above.

Passing the bootstrap tests would be consistent with many of the most appealing and commonly arising inflationary models, with the fewest degrees of freedom, fewest parameters, and smoothest evolution [12]. For example, in Fig. $1, V(\varphi)=m^{2} \varphi^{2}$ corresponds to the open circle (and hence passes the first bootstrap test); $V(\varphi)=\lambda \varphi^{4}$ corresponds to the upper endpoint of the solid curve; the symmetry-breaking (Higgs) potential $V(\varphi)=\Lambda^{4}[1-$ $\left.(\varphi / \mu)^{2}\right]^{2}$ corresponds to the part of the solid curve below the open circle; and the pseudo-Nambu-Goldstone-boson (axion) potential $V(\varphi)=\Lambda^{4}[1 \pm \cos (\varphi / \mu)]$ lies within the shaded region. On the other hand, hybridlike inflation models, including some of the widely discussed proposals motivated by string theory, would not pass the tests [13].

The bootstrap tests have the advantage that they can be performed with forthcoming data. Compare them with the well-known consistency relations for single-field inflation: (i) a hierarchy of relations between the scalar and tensor power spectra [4]; and (ii) a hierarchy of relations between the primordial scalar $N$-point functions [5]. Confirming any of these relations requires measuring either (i) a nonzero value for the tensor spectral index $n_{t}$, or (ii) a non-Gaussian primordial $\mathrm{N}$-point function, both of which will be extremely difficult given the single-field inflationary predictions. Failure (detection of large deviations from the consistency relations) is observationally much easier than confirmation given the limitations of technology and foregrounds. By contrast, with the bootstrap tests, inflation can be precisely tested and confirmed using accessible technology and plausible foregrounds. (The consistency relations might eventually yield additional confirming tests.)

What if the bootstrap tests fail? Of course, there will still be some inflationary models and parameters that agree with the observations, and some that do not. But is there another way of confirming that inflation itself took place? Perhaps there is a generalized framework for inflation that gives rise to a generalized set of bootstrap tests which might still be passed? Interestingly, the two most common generalizations (allowing multiple order parameters $[14,15]$, or replacing the canonical kinetic term $X=\frac{1}{2}(\partial \varphi)^{2}$ by a general function of $X$ [16]) give rise to frameworks that are not testable in our bootstrap sense: the observables $\left\{\Delta_{\mathcal{R}}^{2}, r, n_{s}, \alpha_{s}, \ldots\right\}$ do not intrinsically point to a best guess for both $N_{\text {bef }}\left(k_{*}\right)$ and $N_{\text {aft }}$. An exception is the subclass of single-field $k$-inflation models [16] which only depend on $X$. In this case, a bootstrap test of (1) can be performed if one can also measure the tensor tilt $n_{t}$ (although, as mentioned above, this is likely to be very difficult). An interesting corollary of our analyses is that, in all cases, a direct confirmation of inflation relies on being able to detect cosmic gravitational waves and measure accurately at least $r$.

We thank R. Easther, J. Frieman, W. Kinney, E. Komatsu, and $\mathrm{H}$. Peiris for discussions; and the Princeton Center for Theoretical Science for its role in the development of this Letter. This work was supported by CIFAR (L. B.) and the U.S. Department of Energy Grant DEFG02-91ER40671 (P. J. S.).

[1] A. H. Guth, Phys. Rev. D 23, 347 (1981); A. D. Linde, Phys. Lett. B 108, 389 (1982); A. Albrecht and P. J. Steinhardt, Phys. Rev. Lett. 48, 1220 (1982).

[2] S. Weinberg, Cosmology (Oxford University Press, Oxford, England, 2008).

[3] E. Komatsu et al., Astrophys. J. Suppl. Ser. 180, 330 (2009).

[4] J. E. Lidsey et al., Rev. Mod. Phys. 69, 373 (1997); M. Cortes and A. Liddle, Phys. Rev. D 73, 083523 (2006).

[5] J. M. Maldacena, J. High Energy Phys. 05 (2003) 013; X. Chen, M.-X. Huang, and G. Shiu, Phys. Rev. D 74, 121301 (2006).

[6] D.S. Salopek and J.R. Bond, Phys. Rev. D 42, 3936 (1990); A. R. Liddle, P. Parsons, and J. D. Barrow, Phys. Rev. D 50, 7222 (1994).

[7] W. H. Kinney, Phys. Rev. D 66, 083508 (2002); A. R. Liddle, Phys. Rev. D 68, 103504 (2003); H. V. Peiris and R. Easther, J. Cosmol. Astropart. Phys. 07 (2006) 002; 10 (2006) 017; 07 (2008) 024.

[8] For our purposes, using $H(\varphi)$ has an advantage over the other common alternative $V(\varphi)$. Namely, Eqs. (4)-(6), which play an important role in our analysis, are exact in terms of $H(\varphi)$, whereas the corresponding equations, when expressed in terms of $V(\varphi)$, invoke the slow roll approximation near the end of inflation, where it is expected to break down.

[9] Note that Taylor ordering $\left\{r, n_{s}, \alpha_{s}, \beta_{s}, \ldots\right\}$ is not the order in which these quantities are detected in practice: $n_{s}$ has already been measured, but nonzero values for the remaining quantities (including $r$ and $\alpha_{s}$ ) have not yet been detected [3]. Still, the Taylor ordering is what is relevant for conducting bootstrap tests: for example, until one detects the first observable $r$, none of the bootstrap relations may be confirmed.

[10] R. Easther and H. Peiris, J. Cosmol. Astropart. Phys. 09 (2006) 010

[11] E. Komatsu (private communication).

[12] L. A. Boyle, P. J. Steinhardt, and N. Turok, Phys. Rev. Lett. 96, 111301 (2006).

[13] A. Linde, Phys. Rev. D 49, 748 (1994); D. Baumann and L. McAllister, Phys. Rev. D 75, 123508 (2007); E. Silverstein and A. Westphal, Phys. Rev. D 78, 106003 (2008).

[14] A. D. Linde, Phys. Rev. D 49, 748 (1994).

[15] A. Linde and V. Mukhanov, Phys. Rev. D 56, R535 (1997); D. Lyth and D. Wands, Phys. Lett. B 524, 5 (2002).

[16] C. Armendariz-Picon, T. Damour, and V.F. Mukhanov, Phys. Lett. B 458, 209 (1999). 